\title{
Las bóvedas pétreas nervadas de Galicia: identificación de soluciones
}

\section{The Ribbed Stone Vaults of Galicia: Identifying Some Solutions}

\author{
M. J. Freire-Tellado $^{(*)}$, S. B. Tarrío-Carrodeguas ${ }^{(*)}$
}

\section{RESUMEN}

El artículo presenta los distintos grupos de soluciones de bóvedas pétreas nervadas sobre cuatro apoyos que existen en Galicia, representándolas gráficamente a la misma escala. Complementariamente se incluye también un pequeño grupo de bóvedas sobre ocho apoyos. Para clasificar los ejemplos se ha empleado un sistema basado en los rasgos constructivos de las bóvedas, que tiene en cuenta el número de apoyos de éstas y los distintos tipos de nervios presentes. Este criterio sistemático permite apreciar tanto soluciones que tradicionalmente han pasado desapercibidas como las dimensiones con las éstas que han sido construidas.

Palabras clave: Bóvedas; fábricas; piedra; Galicia; patrimonio.

\section{ABSTRACT}

This article presents the different groups of solutions of ribbed stone vaults on four supports that exist in Galicia and it plots them at the same scale. A small group of vaults on eight supports is also included. In order to classify the samples, a system based on the constructive features of the vaults has been used, taking into account their number of supports and the different types of nerves. The systematic approach used allows determining both the dimensions with which the different types of vaults were built as well as the solutions traditionally overlooked.

Keywords: Vault; masonry; stone; Galicia; heritage.

(*) E.T.S. Arquitectura - Universidad de A Coruña (España).

Persona de contacto/Corresponding author: manuel.freire.tellado@udc.es (M. J. Freire-Tellado)

Cómo citar este artículo/Citation: Freire-Tellado, M. J., Tarrío-Carrodeguas, S. B. (2015). Las bóvedas pétreas nervadas de Galicia: identificación de soluciones. Informes de la Construcción, 67(538): eo83, doi: http://dx.doi.org/10.3989/ic.13.174.

Licencia/License: Salvo indicación contraria, todos los contenidos de la edición electrónica de Informes de la Construcción se distribuyen bajo una licencia de uso y distribución Creative Commons Reconocimiento no Comercial 3.o. España (cc-by-nc). 


\section{JUSTIFICACIÓN}

La lectura de las descripciones de los sistemas de abovedamiento incluidas en los textos sobre los edificios del período provoca a menudo una impresión desalentadora. Demasiadas veces las descripciones se resuelven con términos genéricos como «bóveda ojival», «bóveda estrellada» o, como mucho, haciendo referencia al número de claves, «bóveda de 5 claves». En algunos casos afortunados, la presentación de la bóveda se confía a una imagen, pero no es la norma.

Aunque afortunadamente se han ido publicando excelentes trabajos que reconocen la aportación del abovedamiento a la percepción del espacio y se esfuerzan en representarlo en las plantas de los edificios -en la referencia (1) (2) (3) se incluyen algunos trabajos en el ámbito gallego-, se trata de levantamientos generales orientados fundamentalmente a la descripción de edificios complejos, por lo que resulta necesario completarlos para el análisis de las soluciones de las bóvedas: este trabajo intenta presentar las bóvedas existentes en la comunidad gallega y facilitar su estudio y descripción. En su origen está presente también la razón taxonómica: el contacto con el considerable conjunto de bóvedas existentes en Galicia exige ordenarlas y clasificarlas para proceder a su estudio, para organizar -sistematizar-la información sobre ellas.

Existen dos ideas muy extendidas que conviene revisar. La primera es la creencia de que en la Historia del Arte en Galicia existe una suerte de salto en el tiempo que lleva directamente del románico al barroco casi sin solución de continuidad -véase, por ejemplo, la opinión de Lámpere ${ }^{1}$ expuesta (4)-. La segunda es la identificación de la bóveda pétrea nervada con el estilo gótico - esta idea se trasluce en su frecuente designación como «bóvedas góticas y tardogóticas»- si bien esta última equivalencia está siendo fuertemente contestada en la actualidad (5). De la concatenación de ambas ideas se deduciría que en Galicia no existen apenas bóvedas nervadas de piedra, lo cual es una conclusión totalmente errada: no solo éstas son numerosas en nuestra Comunidad -y con algunos de los ejemplos hispánicos más antiguos, como las de la cripta del Pórtico de la Gloria, las primeras bóvedas nervadas españolas- sino que algunas de ellas son de gran calidad.

El lapso temporal «perdido» corresponde a la etapa gótica y a la renacentista, esto es, al tiempo de asentamiento del Císter y de las órdenes mendicantes, así como de reseñables ampliaciones en los edificios religiosos gallegos -son muy numerosos los claustros cubiertos con bóvedas nervadas en la Comunidad- de fundaciones como el Hospital Real en Santiago de Compostela, así como del permanente foco constructor de la Catedral de Santiago, centrado en la ejecución del claustro y dependencias adyacentes.

Sin embargo el ámbito temporal en el que se desarrolla el estudio es más amplio, extendiéndose más allá de las etapas gótica y renacentista: hay referencias de bóvedas pétreas nervadas mucho más tardías. La bóveda que cubre el tramo de los pies de la iglesia del Monasterio de Samos es una bóveda de doble tercelete datada en el siglo XVIII; las bóvedas de terceletes de las naves del Santuario de Nuestra Señora de los Milagros en Baños de Molgas tienen grabadas en sus claves

\footnotetext{
${ }^{1}$ Lámperez: «Puede decirse que Galicia pasó sin intermedio desde el estilo románico del siglo XII al barroco del XVIII». En el mismo sentido se manifiestan otros autores como Ángel del Castillo o Andrés Rosende.
}

los años de 1753 (nave sur), 1757 (nave norte) y Rivas Quintás (6) acota el periodo entre 1761 y 1769 para la construcción de bóveda de su cimborrio; la profesora Pernas Alonso señala en su Tesis Doctoral (7) que en la clave de la bóveda del cañón de la Escalera de Honor de San Esteban de Ribas de Sil está grabado el año de 1739; Marcos de Vega, en su Trabajo Fin de Máster (8), indica que en el Libro de Obras del Monasterio de San Clodio se recoge la construcción de las bóvedas de las naves colaterales de la iglesia entre 1817 y 1818.

El tránsito de maestros de obras foráneos por la Comunidad dio lugar a que se aplicasen diferentes soluciones de abovedamiento: la actividad constructora en Compostela traerá a Santiago a maestros como Enrique Egas, Juan de Álava, Rodrigo Gil de Hontañón o Alonso de Covarrubias -Martín González (9) cita el encuentro en 1518 de los tres últimos y Juan de Badajoz en Santiago de Compostela, con el fin de debatir técnicamente la propuesta de Álava para el Claustro para la Catedral-. Por Compostela y el resto de Galicia circularon también personajes como Juan de Herrera el Trasmerano, Cornelius de Holanda, Juan Pérez... Tampoco se puede olvidar la circulación de religiosos como consecuencia de las fundaciones del Císter y de las órdenes mendicantes en la Comunidad. Sin embargo conviene aclarar que los pronunciamientos sobre autorías quedan fuera del ámbito de este trabajo.

\section{METODOLOGÍA}

Consistió en la localización, identificación, clasificación, croquización y comprobación dimensional -ya fuese ex novo o de comprobación de trabajos previamente existentes- de las bóvedas nervadas de Galicia. Una característica de este trabajo es su vocación sistemática, dado que se pretende extender el estudio a la totalidad de las soluciones construidas y no solo a los ejemplos más destacados como suele ser habitual. Por razón de extensión, este texto se centra en la presentación de los resultados de esta clasificación, dejando para una segunda parte el análisis de comparado de sus características constructivas.

El proceso de clasificación llevó al diseño del método a aplicar, cuya premisa inicial es que fuese aplicable a simple vista y permitiese la integración de diversos niveles de información dentro de un esquema de conjunto unitario. En este proceso resultó de especial importancia el estudio documental de los levantamientos publicados existentes.

Se ha desarrollado un sistema que atiende a los aspectos constructivos de las bóvedas, a los nervios en cuanto elementos que las conforman y de los que depende su estabilidad, al menos en fase de construcción. Es cierto que las nervaduras de las bóvedas añaden, con el paso del tiempo, aspectos estético-decorativos a la justificación constructiva-estructural inicial, pero no por ello dejan de tener una misión constructiva relevante durante la elevación de la bóveda: antes de cerrar la bóveda colocando la plementería hay que construir la red de nervios que la soportará (10) (11). Y al menos en ese momento la estabilidad del conjunto depende de ellos.

El resultado de este proceso se presenta en la Tabla 1 adjunta, desarrollada en seis niveles de agregación por razones de extensión. La tabla se ciñe exclusivamente a aquellos casos existentes en Galicia si bien la inclusión de ejemplos foráneos permitiría completar la clasificación: sin embargo su consideración se manifiesta en el detalle empleado en ciertos casos -como ocurre en las soluciones de dobles terceletes en ambas direcciones-. 
Tabla 1. Clasificación de las bóvedas nervadas sobre cuatro apoyos existentes en Galicia.

\begin{tabular}{|c|c|c|c|c|c|c|c|}
\hline \multicolumn{7}{|c|}{ BÓVEDAS PÉTREAS NERVADAS SOBRE CUATRO APOYOS } & \multirow{2}{*}{$\begin{array}{c}\text { REF. } \\
\text { Fig. } 1 \\
A 1\end{array}$} \\
\hline \multirow[t]{7}{*}{ Crucería simple } & \multirow[t]{4}{*}{ Sin ligaduras } & Sin combados & & & & & \\
\hline & & \multirow[t]{3}{*}{ Con combados } & Rectos & & & & $\mathrm{B} 1$ \\
\hline & & & \begin{tabular}{|l|} 
Cóncavos completos \\
\end{tabular} & & & & $\mathrm{B}_{2}$ \\
\hline & & & Cuadrifolio & & & & $\mathrm{B}_{3}$ \\
\hline & \multirow[t]{3}{*}{ Con ligaduras } & En 1 dirección & & & & & $\mathrm{A} 2$ \\
\hline & & \multirow[t]{2}{*}{ En 2 direcciones } & Sin tracería & & & & a'1 \\
\hline & & & Con tracería & Cuadrado & & & a'2 \\
\hline \multirow{46}{*}{$\begin{array}{l}\text { Crucería con } \\
\text { terceletes } \\
\text { simples }\end{array}$} & \multirow{3}{*}{$\begin{array}{l}\text { Terceletes en } 1 \\
\text { dirección }\end{array}$} & \multirow[t]{3}{*}{ Con ligadura } & \multirow[t]{2}{*}{ En 1 dirección } & Sin combados & & & $\mathrm{C}_{1}$ \\
\hline & & & & Con combados & & & $\mathrm{C}_{3}$ \\
\hline & & & En 2 direcciones & & & & $\mathrm{C}_{2}$ \\
\hline & Terceletes en 2 & Con ligaduras en 2 & Sin tracería & Con ligadura entre claves & & & D1 \\
\hline & direcciones & direcciones y con & & Con ligadura completa & & & D4 \\
\hline & & tracería en forma de & & Combinada & & & $\mathrm{D}_{5}$ \\
\hline & & & Cuadrado / Rombo rectos & Con ligadura entre claves & & & $\mathrm{F} 1$ \\
\hline & & & entre claves de terceletes & Con ligadura completa & & & $\mathrm{F}_{4}$ \\
\hline & & & Cuadrilátero curvilíneo & Con ligadura entre claves & & & F2 \\
\hline & & & cóncavo entre claves de & Con ligadura completa & & & F3 \\
\hline & & & & Alterna & & & $\mathrm{F}_{5}$ \\
\hline & & & Circunferencia & Entre claves terceletes & & & E1 \\
\hline & & & & Interior parcial & & & E2 \\
\hline & & & Octógono entre & Recto & & & $\mathrm{E}_{3}$ \\
\hline & & & claves de terceletes & Curvilíneo cóncavo & & & $\mathrm{E}_{4}$ \\
\hline & & & $\begin{array}{l}\text { Cuadrifolio entre claves de } \\
\text { terceletes }\end{array}$ & & & & H6 \\
\hline & & & \begin{tabular}{|l|} 
Cuadrilátero curvilíneo \\
\end{tabular} & Con ligadura completa & & & e'1 \\
\hline & & & paralelo al perímetro & Con ligadura entre claves & Combinada con & dobles combados exteriores & $\mathrm{e}^{\prime} 2$ \\
\hline & & & Cuadrifolio exterior & Aislado & & & G1 \\
\hline & & & & $\begin{array}{l}\text { Combinado con } \\
\text { Tracería interior en }\end{array}$ & $\begin{array}{l}\text { Cuadrilátero } \\
\text { curvilíneo cóncavo }\end{array}$ & & * \\
\hline & & & & & Circunferencia & & * \\
\hline & & & & & Óvalo & & * \\
\hline & & & & & Otro & De igual forma & G2 \\
\hline & & & & & cuadrifolio & Diferentes formas & * \\
\hline & & & Cuadrilóbulo (Cruz) exterior & Flameante & Combinada con & Circunferencia interior ligada & G3 \\
\hline & & & & & & Circunferencia desligada & $\mathrm{G} 7$ \\
\hline & & & & Policóncava & Simple & & $*$ \\
\hline & & & & & Combinada con & Circunferencia interior & $\mathrm{G}_{4}$ \\
\hline & & & & & & Octógono interior & * \\
\hline & & & & Multicóncava & Combinada con & Circunferencia interior & $\mathrm{G}_{5}$ \\
\hline & & & & Cruz mixta & Combinada con & Circunferencia interior & G6 \\
\hline & & & $\begin{array}{l}\text { Combados cóncavos entre cl. } \\
\text { ter. bóv. ady. }\end{array}$ & $\begin{array}{l}\text { Ligaduras entre claves y } \\
\text { compl. }\end{array}$ & & & $\begin{array}{l}f^{\prime} 1 \\
f^{\prime} 2 \\
\end{array}$ \\
\hline & & $\begin{array}{l}\text { Ligadura en } 1 \text { dirección } \\
\text { y tracería }\end{array}$ & Cuadrifolio interior & & & & $\mathrm{H}_{7}$ \\
\hline & & $\begin{array}{l}\text { Sin ligaduras pero con } \\
\text { tracería }\end{array}$ & $\begin{array}{l}\text { Entre claves terceletes, con } \\
\text { combados }\end{array}$ & Rectos & $\begin{array}{l}\text { Cuadrado / Rombo } \\
\text { recto }\end{array}$ & & $\begin{array}{l}\mathrm{J}_{1} \\
\mathrm{~J} 2\end{array}$ \\
\hline & & & & & $\begin{array}{l}\text { Octógono irregular } \\
\text { recto }\end{array}$ & & $\mathrm{J} 4$ \\
\hline & & & & & Estrella 4 ptas & & $\mathrm{J}_{5}$ \\
\hline & & & & Curvos & Cuadrilátero cóncavo & & $\begin{array}{l}\mathrm{H} 1 \\
\mathrm{H} 2\end{array}$ \\
\hline & & & & & \begin{tabular}{|l|}
$\begin{array}{l}\text { Circunferencia y } \\
\text { cuadrilátero curvilíneo }\end{array}$ \\
\end{tabular} & & $\mathrm{H}_{3}$ \\
\hline & & & & & Phi griega & & $\mathrm{H}_{4}$ \\
\hline & & & & & Cuadrifolio & Sn más nervios & $\mathrm{H} 9$ \\
\hline & & & & & & Par combados & $\mathrm{H}_{5}$ \\
\hline & & & Exterior a claves terceletes & Cuadrifolio con & Cuadrado recto & & $\mathrm{J} 3$ \\
\hline & & & Sin claves tercelete & En 1 dirección & Cuadrifolio & & $\mathrm{I} 2$ \\
\hline & & & Claves desdobladas & 2 direcciones & Flor y & Circunferencia interior & I1 \\
\hline & & & & & Cuadrifolio y & Otra tracería interior & $\mathrm{I} 3$ \\
\hline & $\begin{array}{l}\text { Terceletes- } \\
\text { combados }\end{array}$ & $\begin{array}{l}\text { Ligadura parcial } \\
\text { longitudinal }\end{array}$ & $\begin{array}{l}\text { Sin claves tercelete } \\
\text { Claves desdobladas }\end{array}$ & 2 direcciones & $\begin{array}{l}\text { Otros nervios } \\
\text { exteriores }\end{array}$ & Circunferencia & $\begin{array}{l}\text { i'1 } \\
\text { i'2 }\end{array}$ \\
\hline
\end{tabular}


Tabla 1. Clasificación de las bóvedas nervadas sobre cuatro apoyos existentes en Galicia. (Continuación.)

\begin{tabular}{|c|c|c|c|c|c|c|}
\hline \multicolumn{6}{|c|}{ BÓVEDAS PÉTREAS NERVADAS SOBRE CUATRO APOYOS } & \multirow{2}{*}{$\begin{array}{c}\text { REF. } \\
\text { Fig. } 1 \\
\text { L1 }\end{array}$} \\
\hline \multirow[t]{7}{*}{\begin{tabular}{l|} 
Crucería con \\
dobles terceletes
\end{tabular}} & \multirow[t]{6}{*}{$\begin{array}{l}\text { Dobles terceletes } \\
\text { en } 1 \text { dirección }\end{array}$} & $\begin{array}{l}\text { Sólo con nervios de } \\
\text { ligadura en ambas } \\
\text { direcciones }\end{array}$ & \multicolumn{3}{|l|}{ Completa } & \\
\hline & & & \multicolumn{3}{|l|}{ Interior a claves de terceletes } & L2 \\
\hline & & \begin{tabular}{|l|} 
Con nervios de \\
ligadura en una \\
dirección y \\
\end{tabular} & $\begin{array}{l}\text { Sin clave tercelete transversal } \\
\text { (claves desdobladas) }\end{array}$ & $\begin{array}{l}\text { Con dobles combados } \\
\text { rectos }\end{array}$ & & L3 \\
\hline & & \multirow{3}{*}{$\begin{array}{l}\text { Con ligaduras en } \\
\text { ambas direcciones } \\
\text { y con tracería en } \\
\text { forma de }\end{array}$} & \multicolumn{3}{|l|}{$\begin{array}{l}\text { Cuadrado recto entre claves de } \\
\text { terceletes interiores }\end{array}$} & L4 \\
\hline & & & Cuadrifolio exterior & \begin{tabular}{|l} 
Combinado con \\
Tracería interior en
\end{tabular} & Circunferencia & L6 \\
\hline & & & Cruz semiexterior & $\begin{array}{l}\text { Combinado con } \\
\text { Tracería interior en }\end{array}$ & Circunferencia & L7 \\
\hline & \begin{tabular}{|l|} 
Dobles terceletes \\
en 2 direcciones
\end{tabular} & $\begin{array}{l}\text { Con ligaduras en } \\
\text { ambas direcciones } \\
\text { y con tracería en } \\
\text { forma de }\end{array}$ & $\begin{array}{l}\text { Cuadrado paralelo al } \\
\text { perímetro por claves de } \\
\text { terceletes interiores }\end{array}$ & \begin{tabular}{|l|} 
Combinado con \\
Tracería interior en
\end{tabular} & Cuadrado & $\mathrm{L}_{5}$ \\
\hline $\begin{array}{l}\text { Crucería sin } \\
\text { terceletes pero } \\
\text { con }\end{array}$ & $\begin{array}{l}\text { Otros nervios en } \\
\text { perímetro }\end{array}$ & $\begin{array}{l}\text { Ligadura completa } \\
\text { ambas direcciones }\end{array}$ & $\begin{array}{l}\text { Cuadrilát. curvilíneo paralelo } \\
\text { al perímetro }\end{array}$ & $\begin{array}{l}\text { Con dobles combados } \\
\text { ext. }\end{array}$ & & e'3 \\
\hline \multirow{2}{*}{\begin{tabular}{l|} 
Ojivos \\
interrumpidos \\
pero con \\
terceletes simples
\end{tabular}} & \multirow[t]{2}{*}{ En 2 direcciones } & \multirow[t]{2}{*}{$\begin{array}{l}\text { Sin ligaduras centrales } \\
\text { y tracería }\end{array}$} & $\begin{array}{l}\text { Cuadrado recto entre claves de } \\
\text { terceletes }\end{array}$ & & & K6 \\
\hline & & & $\begin{array}{l}\text { Circunferencia entre claves de } \\
\text { terceletes }\end{array}$ & & & K7 \\
\hline \multirow[t]{6}{*}{$\begin{array}{l}\text { Sin ojivos pero } \\
\text { con terceletes }\end{array}$} & \multirow[t]{5}{*}{$\begin{array}{l}\text { Terceletes simples } \\
\text { en } 2 \text { direcciones }\end{array}$} & $\begin{array}{l}\text { Con ligadura central } \\
\text { en ambas direcciones }\end{array}$ & Sin otros nervios & & & K2 \\
\hline & & & $\begin{array}{l}\text { Cuadrado recto entre claves de } \\
\text { terceletes }\end{array}$ & & & $\mathrm{K}_{3}$ \\
\hline & & \multirow[t]{3}{*}{$\begin{array}{l}\text { Sin ligaduras pero con } \\
\text { tracería }\end{array}$} & $\begin{array}{l}\text { Cuadrado recto entre claves de } \\
\text { terceletes }\end{array}$ & & & $\mathrm{K} 1$ \\
\hline & & & $\begin{array}{l}\text { Cuadrado curvilíneo entre } \\
\text { claves terceletes }\end{array}$ & & & $\mathrm{K}_{4}$ \\
\hline & & & $\begin{array}{l}\text { Rombo curvilíneo entre claves } \\
\text { terceletes }\end{array}$ & & & $\mathrm{K}_{5}$ \\
\hline & $\begin{array}{l}\text { Terceletes } \\
\text { simples+dobles }\end{array}$ & $\begin{array}{l}\text { Con ligadura central } \\
\text { en ambas direcciones }\end{array}$ & Con otros nervios exteriores & $\begin{array}{l}\text { Con combados entre } \\
\text { claves interiores }\end{array}$ & & K8 \\
\hline
\end{tabular}

La desagregación del tipo da lugar a los subtipos y prosigue en un proceso arborescente que termina en el caso individual. En vez de interrumpir la exposición a un nivel determinado, se optó por la presentación completa de la clasificación, con el fin de facilitar el encaje de nuevas soluciones y de mostrar la descripción sistemática que se deriva del proceso de clasificación efectuado. Ello da lugar a descripciones como «bóveda pétrea nervada sobre cuatro apoyos a base crucería con dobles terceletes en una dirección y simples en la perpendicular, con nervios de ligadura en ambas direcciones y con tracería en forma de cuadrifolio exterior combinado con tracería interior en forma de circunferencia», con un nivel de precisión que no es usual en los textos y susceptible de ser completado fácilmente.

Desde el primer momento se entendió que esta clasificación estaba directamente vinculada con una representación gráfica esquemática de la bóveda con carácter descriptivo: se dibujan los ejes de los nervios, las claves, los apoyos y los bordes, explicando la articulación espacial con el dibujo de una segunda línea. Si bien inicialmente se pensó en el dibujo adimensional del tipo, pronto se sustituyó esta elección por la representación a escala de ejemplos concretos, debido a la relación que existe entre las proporciones generales de una bóveda y la solución particular adoptada para su trazado. Por ello, y para relacionar cada solución con las dimensiones con las que fue construida, se optó por dibujarlas todas a la misma escala.
La Figura 1 se confeccionó con los criterios anteriores. En ella se representan una muestra de las bóvedas estudiadas como síntesis del trabajo desarrollado. Se recogen las distintas clases de bóvedas sobre cuatro apoyos agrupados por filas de acuerdo con los criterios de clasificación expuestos en la Tabla 1 anterior, en cuya última columna bajo el epígrafe $R E F$. Fig 1 - se incluyen las referencias a los ejemplos incluidos en el gráfico -si bien en algún caso se ha omitido el dibujo del ejemplo por considerar que añadía poca información al conjunto-. En aquellos casos en que las bóvedas forman parte de una agrupación, en la ilustración anterior se dibujó un solo tramo de la bóveda, con la excepción de tres casos en los que se representan la duplicación de bóvedas que configuran la solución, puesto que se entendió que la representación de un único tramo no explicaba adecuadamente el conjunto. A la ilustración se la añadió una última fila, con algunas interesantes bóvedas sobre ocho apoyos.

Las bóvedas se identifican señalando la letra que encabeza la fila en la que se encuentra y el número de su posición desde la izquierda -así la bóveda superior izquierda se designa como [A.1]-. En algunos casos particulares se han incluido letras minúsculas seguidas de un apóstrofe, para denotar que se trata de un grupo particular. Como complemento a la lectura del texto, en el Anejo se identifican las bóvedas representadas. 


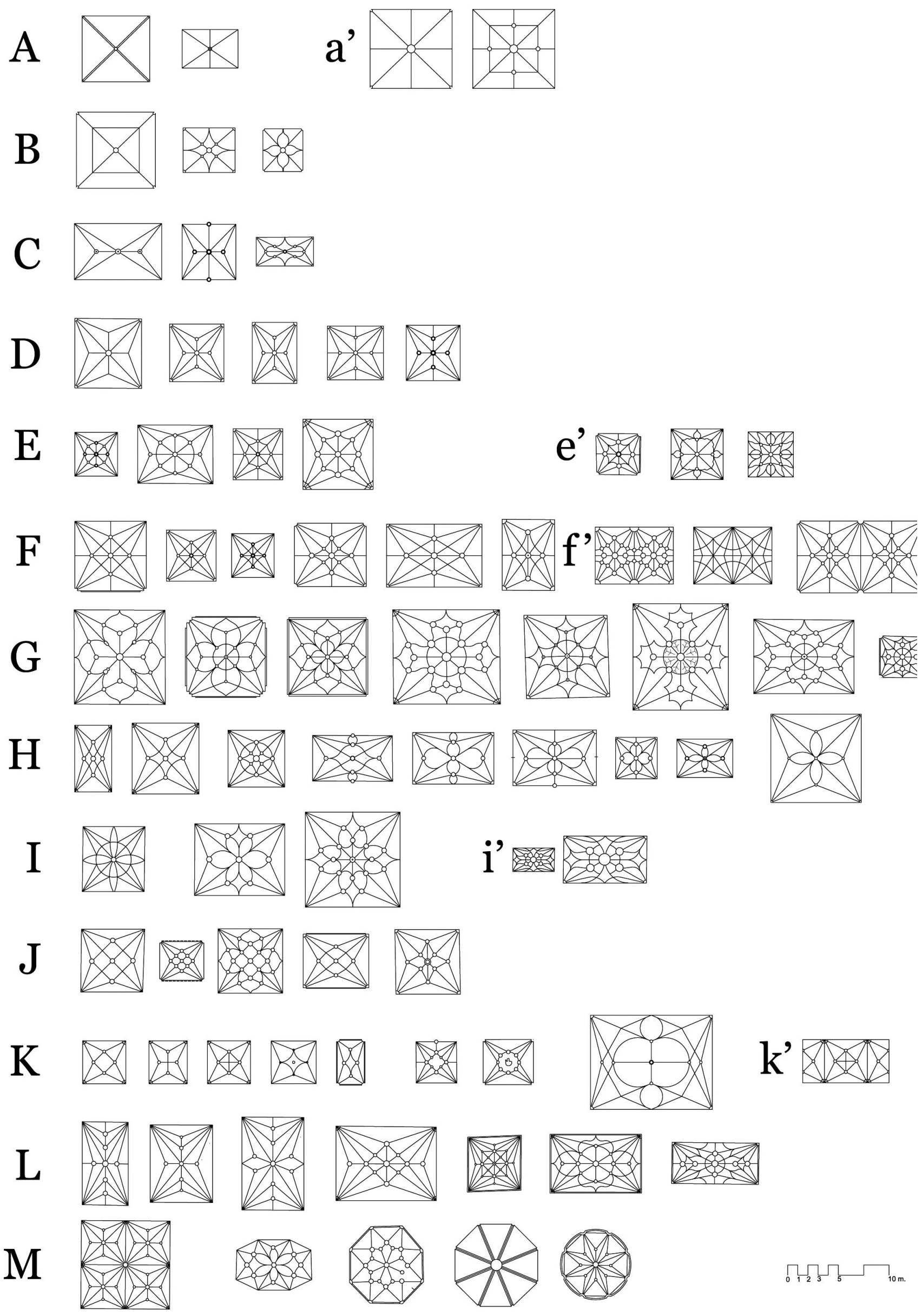

Figura 1. Soluciones de bóvedas nervadas sobre cuatro y ocho apoyos existentes en Galicia. 
El criterio de dibujar casos concretos a la misma escala obliga a decidir qué ejemplo se selecciona cuando las soluciones se repiten. Se ha tendido a incluir la bóveda de mayores dimensiones dentro de cada una de los grupos identificados, considerando que un mayor tamaño suele implicar una mayor capacidad técnica por las dificultades constructivas que introduce. Sin embargo, se ha tratado de huir de una clasificación meramente dimensional, de un criterio de dibujo de «soluciones récord», dado que, además del tamaño, existen otros aspectos a tener en cuenta en la valoración de una bóveda.

Un vistazo a la lámina que ilustra la clasificación realizada permite identificar rápidamente las soluciones más exitosas y el sinnúmero de variaciones que la repetición hizo aflorar, reflejando una suerte de evolución. Se observa también el predominio aplastante de las tracerías con trazados curvos en planta frente a las de trazado rectilíneo en la Comunidad. Pero la Figura 1 muestra además los tamaños en los que se han construido cada una de las soluciones identificadas, permitiendo una comparación de dimensiones inmediata, por lo que resulta muy clarificadora respecto al ámbito de empleo cada solución: no todas han sido aplicadas a todos los tamaños. Esta constatación empírica matiza la extendida idea del proyecto de las bóvedas como una mera cuestión de proporción, adimensional.

Durante la confección del gráfico citado se realizó un agrupamiento de las soluciones en el que pesó el número de variantes representadas. Esta decisión sacrifica ligeramente la pureza de la clasificación en aras a los requerimientos expositivos. Ello llevó a incluir en la misma fila bóvedas con combados rectos y curvos -filas F, J, K, L y M-, aunque desde un punto de vista de adscripción estilística hubiese sido más correcto mantenerlas separadas como se hace en la Tabla 1.

El sistema de trabajo seguido tiene ciertas limitaciones. Por ejemplo, al presentar un módulo de bóveda y no el conjunto, se omiten efectos deslumbrantes alcanzados precisamente gracias a la repetición, como ocurre en la «Sala de las Palmeras» -antigua Sala Capitular del Monasterio cisterciense de Oseira-, y en otros conjuntos de bóvedas palmeadas. Y al presentar exclusivamente el trazado en planta se omiten datos sobre la volumetría de la bóveda, por lo que trazas que en planta pueden resultar muy parecidas correspondan realmente a realidades espaciales marcadamente diferentes. Por ejemplo, pese a la similitud entre las trazas [D.1] y [D.4], su apariencia física difiere notablemente dado que en el primer caso el rampante es redondo y en el segundo es horizontal.

Obviamente no cabe pensar que las soluciones presentadas sean exclusivas de Galicia: al contrario, nos consta no solo la existencia de muchas similares fuera de la Comunidad, sino también -como ya se ha apuntado- la existencia de otros casos que completan y desarrollan la clasificación esbozada. Esto es especialmente claro en las bóvedas con ojivos interrumpidos.

Si bien el número de bóvedas representado rebasa las setenta, realmente son muchas más las estudiadas, dado que detrás de un mismo tipo hay varios casos concretos. Por ello, si bien la cobertura de la muestra estudiada no es completa, representa un porcentaje importante, que se acerca al $100 \%$ en el caso de los edificios más relevantes. Por ello es de esperar que las nuevas aportaciones que aparecerán en el futuro tengan un carácter complementario,-de matiz.

\section{CRITERIOS DE CLASIFICACIÓN}

La clasificación se basa en la descripción de la red de nervios que conforman la bóveda. Se inicia computando el número de apoyos principales de la bóveda en relación a su planta, identificados aquéllos como los puntos de menor cota de ésta; siguiendo este criterio, no se consideran como apoyos las entregas de los arcos de ligadura contra el perímetro. Seguidamente se atiende a los nervios que arrancan desde éstos por orden de importancia -ojivos, terceletes y dobles terceletes- continuando con los nervios de ligadura y de tracería. Se atiende tanto al trazado general de la tracería como a las características de los elementos empleados -rectos o curvos, sus curvaturas así como su perfil-. En el nivel inicial, el estudio se reduce a constatar la existencia o no de cada elemento y a su trazado en planta; en niveles superiores se completa la información subdividiendo cada apartado en función de las características de éste -por ejemplo, clasificando los distintos arcos por su trazado (medio punto, carpanel, a vuelta de cordel...)-. El criterio permite ya en su nivel inicial definir la red de nervios que configura la bóveda en planta, mientras que los siguientes niveles son una aproximación a su volumetría.

El sistema se completa atendiendo a otros rasgos constructivos: la ejecución de los encuentros entre nervios con claves o con cruceros, el nacimiento de los nervios, sobre ménsulas o sobre columnas... No sería difícil añadir otros detalles -por ejemplo, particularidades de las claves, perforadas, labradas, pinjantes...-, pero la prudencia aconsejó no incluirlos para evitar falsear la representación.

La clasificación propuesta no es totalmente unívoca, puesto que ciertos tipos podrían entenderse como subtipos de otros existentes. Priorizar un rasgo determinado tiene un cierto carácter subjetivo. Así la clase I. Claves desdobladas o terceletes interrumpidos, se podría haber entendido como una subclase de la G. Cuadrifolio atendiendo a su traza, pero se ha preferido atender al rasgo constructivo del desdoblamiento de claves -o interrupción de los terceletes- y a la percepción espacial frente al trazado en planta así como a la diferenciación realizada por otros autores: Thunninssen (12) clasifica esta solución como bóveda reticular mientras las anteriores las incluye en las de estrella. La opción se justifica además dentro de la lectura evolutiva que se desprende del gráfico -este tipo deriva de una doble evolución a partir de las filas G y H-.

En algunos casos, la representación se ha forzado un poco. Por ejemplo, en la fila $\mathrm{H}$ se han incluido sendos casos que contienen ligaduras en uno o ambos sentidos, cuando este grupo se caracteriza por la ausencia de éstas. Aquí se ha preferido facilitar la lectura comparada del desarrollo de las denominadas «bóvedas de flor»-aquellas cuya tracería dibuja este elemento (13) - sacrificando a cambio la pureza de la exposición.

Es cierto también que la clasificación que se presenta omite aspectos artísticos muy interesantes, pero se entiende que éstos no son trascendentes desde el punto de vista constructivo -como puede ocurrir con los arcos realizados con dovelas esculpidas-.

La clasificación propuesta no es la única existente en el campo de las bóvedas nervadas: otros autores han propuesto clasificaciones alternativas atendiendo a las proporciones en planta de las bóvedas o de la forma de su rampante. Pese a la indiscutible valía de estas propuestas, la planteada en este artículo presenta la ventaja de su aplicación inmediata, al tiempo que minora los 
problemas provocados por las variaciones de sobre un mismo tipo - una clasificación atendiendo a las proporciones llevaría a repetir trazas similares-. Finalmente, reduce los problemas introducidos por la deformación de las bóvedas, especialmente insidiosos a la hora establecer el perfil del rampante.

\section{DIFICULTADES}

Pese a la supuesta claridad del concepto, bóvedas pétreas nervadas, nos hemos topado con situaciones ambiguas. Así ocurre en las bóvedas de las naves colaterales de la Iglesia de San Jorge en A Coruña -no representada-. Su apariencia general es de bóvedas por arista, pero están construidas con nervios aparejados como tales pero que apenas sobresalen, creando un pequeño resalto que parece pensado para rematar un revoco. En este caso parece dudoso que el nervio pueda resolver la construcción de la bóveda.

Otro aspecto que ha provocado dudas en algunos casos es la distinción entre si una determinada unión de nervios debía ser considerada como clave o como crucero. En algunos casos, especialmente en intersecciones de dos nervios, resultaba complicado discernir si se trataba de una clave de «brazos largos» o ante un crucero de «nervios cortos» dado la ausencia de acceso al intradós de la bóveda. Para su diferenciación se ha recurrido a la longitud de los arranques de los nervios de acuerdo con lo expuesto en referencias como (10).

La dificultad para la diferenciación se ha visto favorecida por la existencia de cruceros con tallas, como ocurre en las bóvedas que cubren los rincones del Claustro Reglar de Celanova, en la nave mayor de Santa María la Mayor [G.1], en la Capilla de las Nieves de la Catedral de Ourense e incluso en el tramo central de la Sacristía de la Catedral de Mondoñedo [F.5].

\section{COMENTARIOS A LAS BÓVEDAS REPRESENTADAS}

Es rigurosamente cierto que durante los períodos gótico y renacentista se inician en esta comunidad menos edificaciones que en los períodos románico y barroco. Por ello las bóvedas nervadas se edifican sobre edificios en construcción, adaptándose a planes previamente establecidos, como ocurre en las Catedrales de Ourense y Tui. Este hecho repercute en las soluciones de arranque de sus nervios, que la menor parte de las veces nacerán desde el suelo y con mucha mayor frecuencia arrancarán a cierta altura, bien desde ménsulas -ménsulas propiamente dichas, capiteles-ménsula, cul-delamp...- bien desde una línea de imposta corrida cuando el abovedamiento se plantea con tiempo suficiente para ello. La introducción del abovedamiento modificando el plan inicial lleva a que los remates perimetrales de las bóvedas se realicen contra muros -que pueden arrancar del suelo o de un arco- con o sin la interposición de un «arco tapajuntas».

Solo en unos pocos casos las bóvedas se prevén desde el inicio, como en las bóvedas de la nave de la Iglesia de San Vicente del Pino en Monforte de Lemos -iniciada en 1539 según se lee en uno de sus contrafuertes- cuyas nervaduras son continuación de columnas que arrancan desde el suelo; en la nave mayor de Santa María la Mayor de Pontevedra -en cuya bóveda del crucero aparece el año de 1559-, en la que se alternan soluciones de arranque y además una imposta interrumpe la continuidad visual nervio-columna; o en la antigua capilla del Palacio de Fonseca, en la que una doble imposta corrida separa el haz de nervios de la bóveda de su columna.

Las bóvedas estudiadas se encuadran bastante bien en las volumetrías generales recogidas por el profesor Palacios (14), si bien, dado que la clasificación de este autor se refiere únicamente a las soluciones más notables, es preciso añadir algún caso más. Así ocurre en las bóvedas sin ojivos o con ellos interrumpidos, cuya parte central aquí se construye totalmente plana. En algún caso además -Claustro del Monasterio de Leiro- la propia bóveda es rebajada, con fajones carpaneles y formeros de medio punto.

La ineludible limitación de espacio lleva a posponer para una segunda parte del artículo los comentarios sobre los tipos de bóvedas representadas y el análisis de sus características constructivas, limitándonos a incluir en estas líneas unos comentarios sobre aquellos casos más particulares -como complemento la referencia (13) ya citada trata los cuadrifolios-.

\section{SOLUCIONES SINGULARES}

Entre los tipos anteriores aparecen algunas soluciones singulares. Un primer grupo de ellas es el formado por bóvedas con ojivos y combados pero carentes de ligaduras y terceletes -fila B-. Son unas bóvedas muy limpias visualmente, pero de reducida dimensión -con lados no superiores a 5,00 m-. Dentro de este grupo se han encontrado dos modelos en la Comunidad:

\section{a) Combados formando un cuadrilátero curvilíneo cóncavo hacia el exterior de la bóveda}

Dicho cuadrilátero une los centros de los lados del perímetro [B.2], y parece una simplificación de las bóvedas del claustro de la catedral compostelana, en la que se renuncian a los terceletes y ligaduras exteriores.

En la mayoría de los casos se construye rebajada y con rampante horizontal, definiendo unas zonas centrales casi completamente planas. Cuenta con ejemplos como son las bóvedas del acceso al Tesoro y Capilla de las Reliquias-Panteón Real en la Catedral de Santiago [B.2], la bóveda del acceso al claustro desde el transepto en esta misma catedral, las bóvedas de la sala adyacente a la Capilla del Palacio de Fonseca en Santiago, la Capilla de San Antón Abade de la Iglesia de Santiago en Betanzos -con un rampante ligeramente curvo- o las bóvedas de los rincones del Claustro de Armenteira, con unas llamativas claves pinjantes (Figura 2). Entre ellas, destacar el elegantísimo desdoblamiento del nervio con el que se genera el cuadrilátero curvilíneo en Santiago (Figura 2).

Las soluciones rebajadas están construidas bien con arcos del perímetro triapuntados (lanceolados) y ojivos rebajados (Catedral de Santiago), con arcos del perímetro carpaneles con ojivos carpaneles también pero muy próximos a arcos deprimidos cóncavos (Armenteira), con arcos apainelados en el perímetro y ojivales carpaneles (Betanzos). Sin embargo, en el Palacio de Fonseca se emplean arcos más usuales, ojivos de medio punto y arcos perimetrales apuntados.

Si bien se podría llegar a pensar que la volumetría de esta bóveda se obtiene al disponer la plementería a la inglesa, 

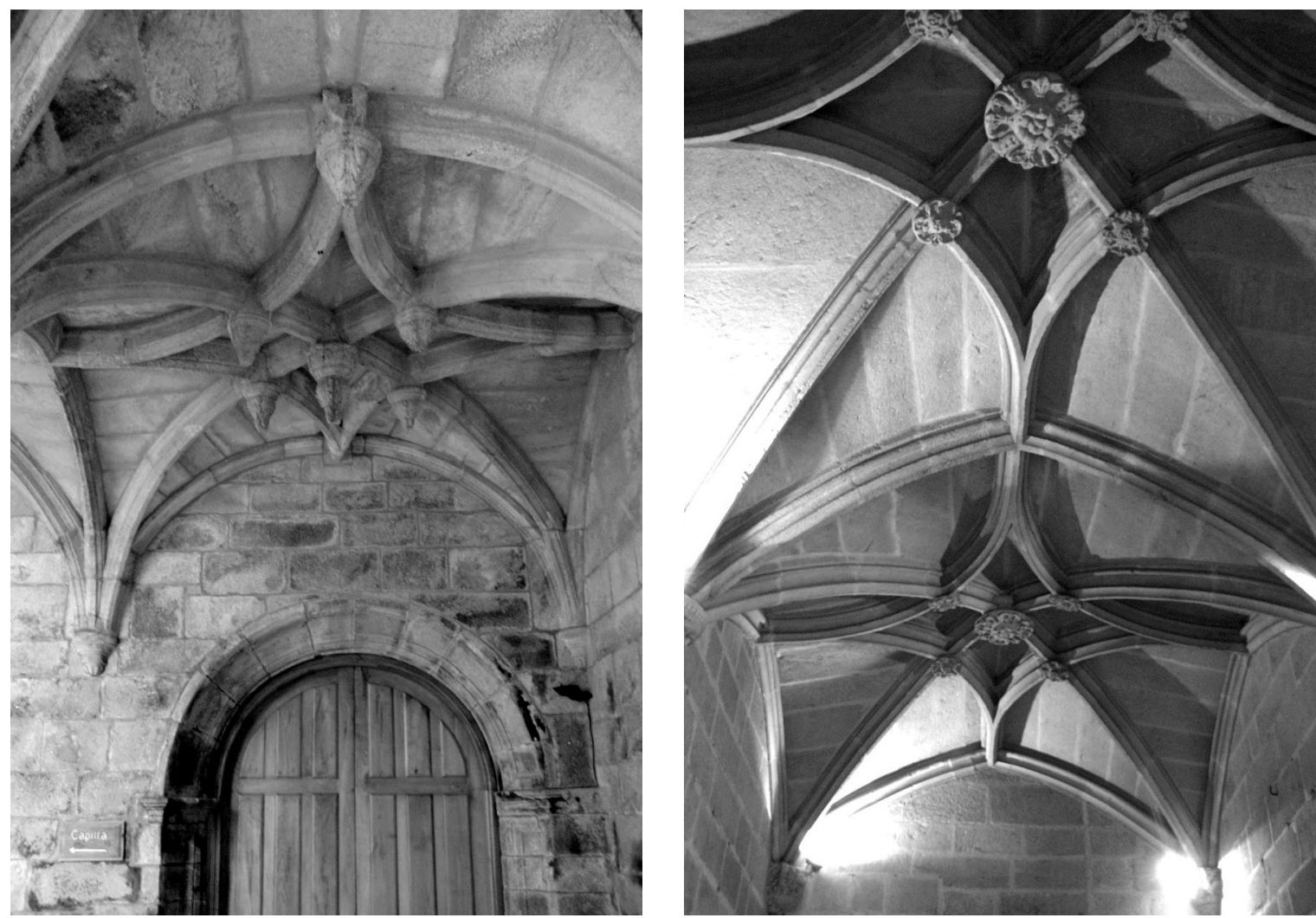

Figura 2. Bóvedas de ojivos y combados cóncavos. Claustro de Armenteira (izq.). Acceso al Tesoro de la Catedral Santiago (dcha.).

ésta se dispone siempre a la francesa en los senos, mientras que la zona central se resuelve con 8 piezas triangulares, disponiendo juntas coincidiendo con los ejes de la bóveda. Se remarca siempre la clave de bóveda, a la que en la que se suele añadir los cuatro encuentros entre los combados y ojivos.

\section{b) Cuadrifolio en forma de flor con remate conopial}

Este tipo está formado por una única bóveda, la de la Capilla de la Purísima de Santa María das Areas en Finisterre [B.3] (Figura 3). Se trata de una bóveda sobre planta rectangular, con ojivos y combados con forma de flor que se extienden a todo lo ancho y largo de la bóveda. La bóveda se construye con una clave tallada y cuatro cruceros en las tangencias entre ojivos y combados. Los nervios nacen de capiteles soportados por columnas encastradas que llegan al suelo. Los combados se entregan contra el perímetro en remates conopiales resueltos de de dos formas diferentes: en pieza única -transversalmente- y en dos piezas paralelas -longitudinalmente-, formando un doble nervio. La plementería se dispone de forma diferente en la parte central y en el perímetro: se coloca a la francesa en el borde -entre ojivos y arcos de cabeza- mientras que en la parte central se colocada transversalmente a los combados del cuadrifolio sobre los que apoya.

Anteriormente se ha señalado como una insuficiencia del sistema de clasificación la desatención a los efectos que produce la repetición de bóvedas. Esto ocurre en la Antesacristía de la Catedral de Santiago de Compostela [f'.1-f'.2]. Es una bóveda de dos tramos, que se considera intermedia entre las bóvedas de terceletes y las de abanico. Que el sistema va más allá de la bóveda de terceletes lo demuestra el énfasis que se da a los apoyos palmeados, especialmente a los centrales, en los que convergen haces de nervios de igual sección -el arco perpiaño es de igual sección que el resto de los nervios-, aunque la plementería no acompañe el giro. Se refuerza la sensación trazando los combados como arcos de circunferencia con centro en los apoyos. Dichos combados no alcanzan el eje de la bóveda, por lo que diferencian la zona central de las zonas de los apoyos -evocando lo que ocurre en las bóvedas del claustro de Convento de San Esteban de Salamanca de Juan de Álava-. Las intersecciones de estos combados con los haces de nervios que nacen de los apoyos se remarcan con labra. Por el contrario, se considera que no llega a ser una bóveda de abanico por la existencia de ligadura transversal uniendo

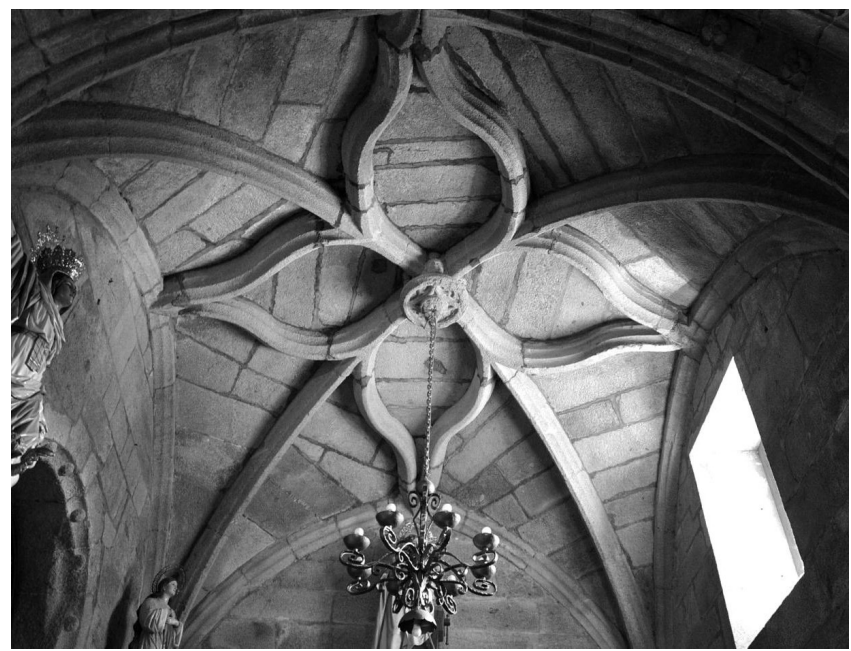

Figura 3. Bóveda de ojivos y cuadrifolio. Capilla de Sta. Ma das Areas, Finisterre. 
las claves de terceletes, por una ligadura longitudinal que no es horizontal y por la valoración de las claves: se sigue priorizando la clave de bóveda y las de terceletes.

La solución presenta una curiosidad en el trazado de los terceletes: en el sentido transversal a la sala, se trazan según la bisectriz del ángulo definido por el ojivo y el arco de cabeza, pero en el longitudinal se trazan enlazando la clave de los terceletes anteriores con los extremos del lado opuesto de la sala -lado menor-.

Finalmente aclarar que se ha tenido que forzar nuevamente la clasificación para colocar la bóveda de la Sacristía de la Colegiata de Xunqueira de Ambía [e'.2] al lado de la bóveda de la Capilla de la Trinidad de Santa María la Mayor [e'.3] -que difícilmente puede ser definida como una bóveda con terceletes- dado que algunas referencias bibliográficas las relacionan (15). Ambas bóvedas tienen un tamaño similar, son bóvedas de ojivos con una decoración de combados de curvatura múltiple en planta que dibujan un cuadrado curvilíneo y ambas completan su tracería con cuatro hojas ubicadas en el centro de las caras del cuadrado anterior. Pero ahí acaban los parecidos: la bóveda de Xunqueira es claramente una bóveda de ojivos, terceletes y cinco sencillas claves, con ligaduras entre las claves de terceletes, rampante llano con la tracería ya descrita mientras que en la capilla de la Trinidad no existen los terceletes convencionales; cada uno ha sido sustituido por un sistema de doble terceletes-combados, que no arrancan del vértice de la bóveda sino de punto intermedio de los arcos de perímetro y llegan tanto a la clave de tercelete como a una clave intermedia en el ojivo. Las claves son aquí 9 -poderosas, labradas y cromadas-, las ligaduras son continuas a lo largo de toda la bóveda con rampante curvo -quizás buscando su colaboración en la estabilidad dado el trato recibido por el tercelete- y la forma de las hojas de decoración es diferente, insinuando apenas el conopio. La plementería de Pontevedra está revestida y pintada de azul claro; la de Xunqueira no parece haber sido concebida para revestir y muestra el granito en el que está ejecutada.

\section{CONCLUSIONES}

- La clasificación propuesta, recogida en la Tabla 1, permite una designación sistemática de las bóvedas, poniendo de manifiesto las variantes y soluciones singulares, por lo que aconseja limitar el empleo de términos generales -como bóvedas estrelladas- a aquellos casos que carezcan de otra designación más precisa.

- La ilustración que se acompaña (Figura 1) proporciona una visión general de las bóvedas sobre cuatro apoyos existentes en Galicia. Evidencia la existencia de un importante número de bóvedas, recoge las variantes existentes -entre los cuales aparecen algunas variantes propias-, e informa de las dimensiones con las que han sido construido, que no son muy grandes salvo contadas excepciones.

- Las bóvedas estudiadas fueron construidas durante los períodos gótico y renacentista, pero existen también ejemplos barrocos, del siglo XVIII e incluso principios del XIX, tal y como se expone en la Justificación.

- El dibujo a escala de los distintos ejemplos pone de manifiesto que no todas las soluciones se han construidos en los distintos tamaños. Esta constatación matiza la idea del proyecto proporcional de las bóvedas.

- La representación acota las relaciones entre las distintas bóvedas del panorama construido, dado que las semejan- zas en algunos casos se limitan a similitudes parciales entre las tracerías.

- La representación evidencia algunas características de las bóvedas: la mayoría de ellas nace de ménsulas, las bóvedas con tracería curva es mucho más frecuente que la recta.

- El trabajo ha puesto de manifiesto la existencia de algunas trazas construidos con distintos tipos de, lo cual resulta en volumetrías muy diferentes. También se ha constatada la construcción de determinadas soluciones con claves y con cruceros.

\section{CLASIFICACIÓN: LISTADO DE BÓVEDAS REPRESENTADAS}

En este apartado se identifican las bóvedas representadas en la ilustración (Figura 1).

A. Bóveda de crucería simple.

A.1. Pórtico acceso de la Catedral de Tui, Pontevedra.

A.2. Bóveda del primer tramo del Presbiterio de la Iglesia del Convento de la Merced en Sarria, Lugo.

a'. Bóvedas de crucería con ligaduras en ambas direcciones. a'.1. Capilla del Pilar de la Catedral de Lugo.

a'.2. Capilla del Santísimo de la Catedral de Tui (con 'combados rectos').

B. Bóvedas de crucería sin ligaduras pero con combados.

B.1. Capilla Mayor de Santa Columba de Rianxo, A Coruña.

B.2. Catedral de Santiago: Bóveda del acceso al Tesoro y Panteón Real.

B.3. Santa María das Areas, Finisterre, A Coruña: Bóveda de la Capilla de la Purísima (fundada en 1496).

C. Bóvedas de crucería con terceletes en una dirección.

C.1. Monasterio de San Estevo de Ribas do Sil, Ourense: Nave de la Iglesia.

C.2. Panda Este del claustro del Monasterio de Poio, Pontevedra.

C.3. Bóveda menor del Tesoro de la Catedral de Santiago.

D. Bóvedas de crucería con terceletes en ambas direcciones y ligaduras.

D.1. Capilla Mayor de Santa Mariña Dozó en Cambados, Pontevedra.

D.2. San Miguel dos Agros, Santiago de Compostela.

D.3. Claustro de los Obispos del Monasterio de Ribas do Sil, Ourense -panda O, bóveda mayor-.

D.4. Claustro del Monasterio de Celanova, Ourense - pandas $\mathrm{N}, \mathrm{S}$ y O-.

D.5. Claustro del Monasterio de Poio, Pontevedra -pandas $\mathrm{N}, \mathrm{S}$ y O-.

E. Bóvedas de crucería con terceletes en 2 direcciones, ligaduras y combados aprox. circulares.

E.1. Capilla de la Purísima de Santa María la Mayor de Pontevedra.

E.2. Tesoro de la Catedral de Santiago.

E.3. Capilla de San Cayetano de $\mathrm{N}^{\mathrm{a}}$ Sra. del Camino, Betanzos, A Coruña.

E.4. Cañón de la Escalera de Honor del Monasterio de San Esteban de Ribas do Sil, Ourense.

e'.1. Bóveda Lateral (lado del Evangelio) del Pórtico del Paraíso de la Catedral de Ourense.

e’.2 Bóveda de la Sacristía de la Iglesia de Xunqueira de Ambía, Ourense.

e'.3. Capilla de la Trinidad, Iglesia de Santa María la Mayor en Pontevedra

F. Bóvedas de crucería con terceletes en ambas direcciones, ligaduras y combados formando un cuadrilátero entre claves de terceletes. 
F.1. Bóveda del brazo sur del transepto de la Iglesia del Monasterio de Santa María de Melón, Pontevedra.

F.2. Capilla de San Antón Na Sra. del Camino, Betanzos.

F.3. Capilla del Pilar de Santa María la Mayor de Pontevedra.

F.4. Sala -antigua capilla- del Palacio de Fonseca.

F.5. Sacristía de la Catedral de Mondoñedo, Lugo -tramo central-.

F.6. Capilla lateral de la Iglesia de San Vicente del Pino, Monforte de Lemos, Lugo.

f'.1. Antesacristía de la Catedral de Santiago.

f'.2. nervios de la misma bóveda .

f'.3. Sala -antigua capilla- del Palacio de Fonseca -ambas bóvedas-.

G. Bóvedas de crucería con terceletes en ambas direcciones, ligaduras interiores entre claves y tracería en forma de cuadrilóbulos.

G.1. Nave -crucero- de la Iglesia de Santa María la Mayor en Pontevedra.

G.2. Bóveda del crucero de la Iglesia del Monasterio de Santa María de Melón, Pontevedra.

G.3. Bóveda del crucero de Esteban de Ribas do Sil, Ourense.

G.4. Sacristía de la Catedral de Santiago de Compostela.

G.5. Capilla Mayor de la Iglesia de San Francisco de Noia, A Coruña.

G.6. Capilla de las Reliquias - Panteón Real de la Catedral de Santiago de Compostela.

G.7. Nave de la Iglesia de San Vicente del Pino, Monforte de Lemos, Lugo.

G.8. Bóveda Lateral (lado de la Epístola) del Pórtico del Paraíso de la Catedral de Ourense.

H. Bóvedas de crucería con terceletes en ambas direcciones, sin ligaduras pero con combados curvos uniendo claves de terceletes.

H.1. Nave colateral de la Iglesia del Monasterio de San Clodio de Leiro, Ourense.

H.2. Nave central de la iglesia anterior.

H.3. Capilla lateral de San Francisco de Noia, A Coruña.

H.4. Coro Alto de la iglesia del Monasterio de Santa María de Oseira, Ourense.

H.5. Refectorio del Monasterio de Oia.

H.6. Bóveda del primer tramo del presbiterio de la Iglesia de $\mathrm{N}^{\mathrm{a}}$ Sra del Camino, Betanzos, A Coruña.

H.7. Claustro Reglar del Monasterio de Monfero, A Coruña.

H.8. Bóveda del Sotocoro de la Iglesia del Monasterio de Oia.

H.9. Cañón de la Escalera Parador de Baiona, Pontevedra -antigua Capilla Mayor de la iglesia del monasterio de San Francisco-.

I. Bóvedas de crucería con terceletes no concurrentes entre sí en una clave, sin ligaduras pero con combados (clave de terceletes desdoblada en dos subclaves tercelete-combados).

I.1. Crucero de la Catedral de Tui, Pontevedra.

I.2. Refectorio del Monasterio de Santa María de Oseira, Ourense.

I.3. Presbiterio de la Iglesia de San Vicente del Pino, Monforte de Lemos, Lugo. i’.1. Capilla de San Miguel, Santa María la Mayor, Pontevedra.

i'.2. Idem a escala doble debido a su tamaño.

J. Bóvedas de crucería con terceletes en ambas direcciones, sin ligaduras pero con combados rectos uniendo claves de terceletes.

J.1. Portería del Monasterio de Monfero, A Coruña.

J.2. Claustro de las Procesiones del Monasterio de Montederramo, Ourense.

J.3. Presbiterio de la iglesia de Guimarei, Lugo.

J.4. Cañón de la escalera entre claustros de S. Clodio de Leiro, Ourense.

J.5. Estancia entre claustros del Monasterio de Montederramo, Ourense.

K. Bóvedas de terceletes sin ojivos -tipo alemán- o con ellos interrumpidos.

K.1. Rincón del Claustro de la Portería del Monasterio de San Clodio de Leiro.

K.2. Claustro de la Portería del Monasterio de San Clodio de Leiro.

K.3. Sala de paso del Monasterio de San Clodio de Leiro

K.4 Claustro Reglar del Monasterio de Oia.

K.5. Nave colateral de la Iglesia del Monasterio de de Santa María Oseira, Ourense.

K.6. Rincón del Claustro las Procesiones del Monasterio de Poio.

K.7. Capilla de Alba de la Iglesia de Santiago de Puebla del Deán, A Coruña .

K.8. Antigua Sala Capitular del Monasterio de Oia, Pontevedra.

L. Bóvedas con oijvos y dobles terceletes.

L.1. Naves colaterales de la iglesia de Santa María la Mayor, Pontevedra.

L.2. Presbiterio de la Iglesia de Puebla del Caramiñal, A Coruña.

L.3. Capilla Mayor de San Pedro de Melide -iglesia del antiguo Convento de Franciscanos Terciarios Sancti Spiritus, A Coruña.

L.4. Sotocoro de la Iglesia de San Vicente del Pino, Monforte de Lemos, Lugo.

L.5. Capilla de San Juan Bautista de la Catedral de Ourense.

L.6. Pórtico Norte de la Catedral de Lugo.

L.7. Bóveda Central del Pórtico del Paraíso de la Catedral de Ourense.

M. Bóvedas de 8 apoyos.

M.1. Bóveda de la linterna del Hospital Real de Santiago de Compostela -hoy Hostal de los Reyes Católicos-.

M.2. Bóveda de la Escalera de los Obispos del Monasterio de de Santa María Oseira, Ourense.

M.3. Bóveda del cimborrio de la Catedral de Ourense.

M.4. Bóveda del crucero de la Catedral de Santiago de Compostela.

M.5. Bóveda del cimborrio del Santuario de Nuestra Señora de los Milagros en Baños de Molgas, Ourense.

Las referencias [E.4] y [J.4] se han dibujado a partir de los levantamientos incluidos en la tesis de $\mathrm{D}^{\mathrm{a}}$ Inés Pernas (4).

\section{REFERENCIAS}

(1) Franco, J.A., Tarrio, S.B. (directores). (1999). Las Catedrales de Galicia. Descripción gráfica. Santiago de Compostela: U.d.C. - Xunta de Galicia Ed.

(2) Franco, J.A., Tarrio, S.B. (directores). (2002). Monasterios y Conventos de Galicia. Descripción gráfica de los declarados monumentos. Santiago de Compostela: U.d.C. - Xunta de Galicia Ed.

(3) Franco, J.A., Tarrio, S.B. (directores). (2009). Iglesias de los Monasterios y Conventos de Galicia. Descripción gráfica de las declaradas monumento. Santiago de Compostela: U.d.C. - Xunta de Galicia Ed.

(4) Lampérez, V. (1908). Historia de la Arquitectura cristiana española en la Edad Media. Madrid: Reprod. facs. 
(5) Palacios, J. C. (2009). La Cantería Medieval. La Construcción de la bóveda gótica española, pp.75-76. Madrid: Munilla -Lería.

(6) Rivas Quintas, E. (2002). Santuario de Nuestra Señora de los Milagros Baños de Molgas, Ourense, p. 33. León: Edilesa.

(7) Pernas, M. I. (2011). Escaleras de piedra en los conjuntos monásticos de la provincia de Ourense entre los siglos XVI al XVIII. Análisis Gráfico (Tesis Doctoral). A Coruña: Universidade da Coruña.

(8) Marcos, L. (2013). San Clodio de Leiro: Origen y evolución constructiva (Trabajo Fin de Maestría no publicado). A Coruña: Universidade da Coruña.

(9) Martín, J. (1977). El Renacimiento. En AA.VV. IX Centenario de la Catedral de Santiago. Año Santo de 1976, (pp. 289328). Santiago de Compostela: Servicio de Publicaciones de la Caja de Ahorros de Santiago.

(10) Palacios, J. C., Martín, R. (2009). La construcción de una bóveda de crucería en la Escuela Técnica Superior de Arquitectura de Madrid. Informes de la Construcción, 61(515): 49-58, doi: http://dx.doi.org/10.3989/ic.08.058.

(11) Rabasa, E. (2005, 27-29 de enero). Construcción de una bóveda de crucería en el centro de oficios de León. En Actas del Cuarto Congreso Nacional de Historia de la Construcción, (pp. 909-918). Cádiz: Instituto Juan de Herrera.

(12) Thunninssen, H.J.W. (1950). Bóvedas: su construcción y empleo en la arquitectura, Láminas 61 y 70. Amsterdan: N.V. Wed, Ahrend \& Zoon. -Edición española por Huerta, S., García, R. (2012). Madrid: Instituto Juan de Herrera-.

(13) Freire, M. (2011, 26-29 de octubre). Flores en los Techos de Galicia: la tracería de las bóvedas nervadas. En Actas del Séptimo Congreso Nacional de Historia de la Construcción, (pp. 421-430). Compostela: Instituto Juan de Herrera.

(14) Palacios, J. C. (2009). La Cantería Medieval. La Construcción de la bóveda gótica española, pp. 117-120. Madrid: Munilla-Lería.

(15) Fernández, B. (2004). Santa María la Mayor. Una iglesia parroquial, pp. 68-69. Santiago de Compostela: Xunta de Galicia. 